\title{
INTRINSIC FORMALITY AND CERTAIN TYPES OF ALGEBRAS
}

\author{
GREGORY LUPTON
}

\begin{abstract}
In this paper, a type of algebra is introduced and studied from a rational homotopy point of view, using differential graded Lie algebras. The main aim of the paper is to establish whether or not such an algebra is the rational cohomology algebra of a unique rational homotopy type of spaces. That is, in the language of rational homotopy, whether or not such an algebra is intrinsically formal. Examples are given which show that, in general, this is not so-7.8 and 7.9. However, whilst it is true that not all such algebras are intrinsically formal, some of them are. The main results of this paper show a certain class of these algebras to be intrinsically formal-Theorem 2 (6.1); and a second, different type of algebra also to be intrinsically formal-Theorem 1 (5.2), which type of algebra overlaps with the first type in many examples of interest. Examples are given in $\S 7$.
\end{abstract}

\section{Preliminaries, Notation and an aCKNOWLedgment}

In [D-Gr-Mo-Su] it is proven that compact Kähler manifolds are formal spaces. From the rational homotopy point of view, this means that compact Kähler manifolds are particularly interesting as examples. This paper is concerned with a more algebraic approach than that of [D-Gr-Mo-Su]. The cohomological properties of compact Kähler manifolds are abstracted out-see 3.1 -and algebras satisfying these properties are studied, with a particular view to deciding whether or not such algebras are intrinsically formal-see 2.1.

The main results of this paper are Theorems 1 and 2-5.2 and 6.1; many examples are also given in $\S 7$. Also in $\S 7$, examples are given of algebras that are not intrinsically formal, and yet satisfy the properties referred to above-see 7.8, 7.9. Rather than give a step by step breakdown of the paper here, I have put one or two sentences heading each section. These section headings can be read in order, at this stage, so as to give an overview of the contents of the paper.

For the fundamentals of rational homotopy theory, the basic references are, in alphabetical order, [Bou-Gu, Gr-Mo, $\mathrm{H}_{1}, \mathrm{Q}, \mathrm{Su}_{1}$, and $\mathrm{Su}_{2}$ ]. This paper will, throughout, only be concerned with the "simply connected with rational homology of finite type" case. For references which give all the results needed for this

Received by the editors July 29, 1987.

1980 Mathematics Subject Classification (1985 Revision). Primary 55P62, 55P15; Secondary $14 \mathrm{M} 10,53 \mathrm{C} 55$.

Key words and phrases. Rational homotopy, intrinsic formality, Kähler manifolds. 
paper in an accessible way, although in more generality than is needed here, see $\left[\mathrm{N}_{2}, \mathrm{~N}-\mathrm{Mi}\right]$.

As for notation, the basic terminology-(DG) algebras, (DG) Lie algebras, minimal algebras, minimal Lie algebras-follows that of [Q, p. 209 and Appendix $\mathrm{B} ; \mathrm{N}_{2}, \mathrm{~N}-\mathrm{Mi}$. In particular, for a graded vector space $V, \mathbf{L}(V)$ will mean the free graded Lie algebra on $V ; \Lambda(V)$ will mean the free graded commutative algebra on $V ; s^{-1} V$ will mean the desuspension of $V$-i.e., $\left(s^{-1} V\right)^{i}=V^{i+1}$ and take $s^{-1} V^{0}=0$. Lie algebras will always be 0 -connected$L^{0}=0$ - and of finite type-finite dimension in each degree. DG algebras will normally, but not necessarily, be of finite type; and cohomology algebras will always be of finite type. Apart from in the second paragraph of $\S 2$, a space will mean a 1-connected topological space with rational homology of finite type, and the homology or cohomology of a space will always mean singular with rational coefficients. A rational homotopy type will mean an equivalence class of spaces under rational homotopy equivalence [Q, p. 208]. By a space, or rational homotopy type, or DG algebra realising an algebra will be meant, respectively, that the space, any representative of the rational homotopy type, or the DG algebra has cohomology isomorphic to that algebra. Finally, a quadratic differential on a free Lie algebra-the only context, in this paper, where such a differential will be encountered-will mean a differential $\partial: \mathbf{L}(V) \rightarrow \mathbf{L}(V)$ such that $\partial(V) \subset[V, V]$. The notation $\partial$ will invariably mean a quadratic differential.

The main ideas of this paper form part of my Ph. D. thesis [Lu], which was written under the supervision of Professor Elmer Rees. It was Elmer who suggested first that I work in rational homotopy theory, then that I consider the specific problem which resulted in my thesis; and I am happy to be able to thank him for all the help and encouragement he has given me. Thank you Elmer.

\section{INTRODUCTION}

In this section the intrinsic formality problem is introduced, and a brief summary of the known results is given. Also some features of the DG Lie algebra approach to intrinsic formality are recalled.

The problem that lies at the heart of this paper is a seminal one in algebraic topology: given a graded algebra, can it be realised as the cohomology algebrawith appropriate coefficients - of some space; and if so, how many homotopy types share that algebra as their cohomology? If you choose to work in rational homotopy, this problem is simplified somewhat. In fact, the first part of the problem collapses. That is, given a 1-connected graded algebra over the rationals, of finite type, there exists a rational homotopy type that has that algebra as its rational cohomology algebra [Q, p. 210]. The second part of the problem is known as the intrinsic formality problem.

2.1. Definition. Let $H$ be a 1-connected graded algebra over $\mathbb{Q}$ of finite type. $H$ is intrinsically formal if there is only one rational homotopy type realising $H$. 
As the words would suggest, intrinsic formality is closely related to the concept of formality. Recall that a minimal DG algebra, $(M, d)$, is formal if there exists a cohomology isomorphism $\psi: M, d \rightarrow H^{*}(M, d)$; and a space is formal if its minimal model is. This definition can be explained as follows. Amongst other things, Sullivan constructed an equivalence between the rational homotopy category of 1-connected spaces having rational cohomology of finite type and the homotopy category of associative, commutative, DG algebras over $\mathbb{Q}$ having 1-connected homology of finite type $\left[\mathrm{Su}_{2}\right]$. Thus, any rational homotopy type can be regarded as a homotopy type of DG algebras. A specific representative for this homotopy type of algebras was also constructed, the minimal model of a space. Now, the rational cohomology of a space $X, H^{*}(X ; \mathbb{Q})$, can be regarded as a DG algebra with trivial differential; but in general it will not be of the same homotopy type as the minimal model of $X$. If it is, then $X$ is formal. Since all DG algebras in a homotopy type share the same minimal model up to isomorphism, this is what is meant by the minimal model being a formal consequence of the cohomology $H^{*}(X ; \mathbb{Q})$. That is, the cohomology can be used to build a minimal model of $X$. See also the definitions and conditions equivalent to formality in [D-Gr-Mo-Su, N-Mi].

Formality and intrinsic formality are related as follows. If a space $X$ is formal, then $H^{*}(X ; \mathbb{Q})$ need not be intrinsically formal. However, if an algebra $H$ is intrinsically formal, then the rational homotopy type realising $H$ must be formal. This follows since every algebra is realised by some formal DG algebra-specifically the minimal model of $H$; and if $H$ is intrinsically formal, any other DG algebra realising $H$ must be homotopic to this formal one and hence formal. In fact, the latter provides the following

2.2. Equivalent definition. An algebra $H$ is intrinsically formal if every space realising $H$ is formal.

Thus, intrinsic formality provides a means of showing formality in some cases. Indeed, the original motivation for this work came from the following. In [D-Gr-Mo-Su] it is shown that compact Kähler manifolds are formal. Actually, it is proved there that compact Kähler manifolds are formal over the reals, and subsequently theorems of descent have been proved, which imply formality over the rationals [H-St, Corollary 6.9; N-Mi, Corollary 5.4]. Now, it is known that compact Kähler manifolds have an interesting rational cohomology algebra structure, given by the "hard Lefschetz theorem"-see 3.1. Thus it is natural to ask whether or not this structure forces the rational cohomology algebras of compact Kähler manifolds to be intrinsically formal. See also [Bab, p. 249]. This paper should be seen as beginning the analysis of this question.

The concept of intrinsic formality first appeared as a remark in [ $\mathrm{Su}_{2}$, p. 317]. Examples were also given there. Let $H$ be isomorphic to

$$
\mathbb{Q}\left[x_{1}, \ldots, x_{n}\right] /\left(R_{1}, \ldots, R_{m}\right),
$$

where $\left\{R_{1}, \ldots, R_{m}\right\}$ is a regular sequence in $\mathbb{Q}\left[x_{1}, \ldots, x_{n}\right]$. Then $H$ is 
intrinsically formal. Work by a number of authors extending Quillen's work on rational homotopy [Ba-Le, $\mathrm{N}_{2}, \mathrm{~N}-\mathrm{Mi}$ ] meant that problems concerning minimal models, such as formality and intrinsic formality, could be approached by DG Lie algebra methods. In [N-Mi], DG Lie algebras were used to prove that all 1-connected Poincare duality algebras of dimension at most 6 are intrinsically formal. This result was subsequently generalised in $\left[\mathrm{Mi}_{2}\right]$, where it was proved that all $(k-1)$-connected Poincare duality algebras of dimension at most $4 k-2$ are intrinsically formal. In addition, several authors have proved, using either DG algebras [H-St], or DG Lie algebras $[\mathrm{N}-\mathrm{Mi}]$, that all $(k-1)$-connected algebras of dimension at most $3 k-2$ are intrinsically formal. It has also been proven that the rational cohomology algebra of a stunted infinite complex projective space, $H^{*}\left(\mathbb{C} P^{\infty} / \mathbb{C} P^{n} ; \mathbb{Q}\right)$, is intrinsically formal [Ta].

The results of this paper are proved using DG Lie algebra methods. These were adopted because DG Lie algebras provide a useful means of approach to the intrinsic formality problem-see 4.3 . The basic facts concerning DG Lie algebras in rational homotopy theory are contained in [Q, $\left.\mathrm{N}_{2}, \mathrm{~N}-\mathrm{Mi}\right]$. For convenience, some facts are recalled here. First, rational homotopy types are equivalent to homotopy types of DG Lie algebras [Q, Theorem 1]. In [Ba-Le, $\left.\mathrm{Mi}_{1}\right]$ (see $\left[\mathrm{Mi}_{2}\right]$ ), minimal models for DG Lie algebras were introduced, and a relatively simple description of the DG Lie algebra minimal model for a rational homotopy type was given. This is as follows. Let $X$ represent the rational homotopy type. Then on $\mathbf{L}\left(s^{-1} H_{*}(X ; \mathbb{Q})\right)$, the free Lie algebra on the desuspension of the rational homology of $X$, there is some decomposable differential $d$, such that $\mathbf{L}\left(s^{-1} H_{*}(X ; \mathbb{Q}), d\right)$ is the minimal model of $X$. Furthermore, for each $X, d$ splits as the sum of $\partial_{\Delta}+d_{I}$, where $\partial_{\Delta}$ is the quadratic part of $d$, and can be read off from the algebra structure-in fact from the dual coalgebra structure- as

$$
\partial_{\Delta}\left(s^{-1} c\right)=-\sum_{i<j}(-1)^{\left|c_{i}\right|}\left[s^{-1} c_{i}, s^{-1} c_{j}\right]-\frac{1}{2} \sum_{i}\left[s^{-1} c_{i}, s^{-1} c_{i}\right],
$$

if $\Delta(c)=c \otimes 1+1 \otimes c+\sum_{i<j}\left\{c_{i} \otimes c_{j}+(-1)^{\left|c_{i}\right|\left|c_{j}\right|} c_{j} \otimes c_{i}\right\}+\sum_{i} c_{i} \otimes c_{i}$ in the coalgebra $H_{*}(X ; \mathbb{Q})$. Note that it is always possible to write $\Delta(c)$ in the above form since $H_{*}(X ; \mathbb{Q})$ is commutative, which also implies that no $c_{i} \otimes c_{i}$ terms for which $\left|c_{i}\right|$ is odd appear in the expression for $\Delta(c)$. In the special case when a DG Lie algebra minimal model of a graded algebra, $H$, has to be built-or equivalently, a DG Lie algebra minimal model of a formal space-then the above formula determines this minimal model, which is $\mathbf{L}\left(s^{-1} H_{*}, \partial_{\Delta}\right)$. This notation will be adopted throughout the paper.

Remark. In view of the fact that DG Lie algebras are often perceived as more obscure, less "natural" than DG algebras, it is perhaps worthwhile noting that each type of object has its own advantages, depending on how the initial information is given. As just one example of this, consider a finite graded algebra $H$. The DG Lie algebra minimal model of $H$ has a finite presentation; whereas, 
in general, this is very far from the case for the DG algebra minimal model of such an $H$. See also [Le-Si].

\section{LEFSCHETZ ALGEBRAS}

In this section a type of graded algebra is introduced, called a Lefschetz alge$b r a$, the analysis of which is a central concern of the paper. Examples are given, together with ways of constructing new Lefschetz algebras from old. Also, general facts and constructions concerning these algebras are established. Particular cases are analysed in later sections.

3.1. Definition. Let $H$ be a graded algebra of even dimension $2 n$. $H$ is a Lefschetz algebra if

1. $H$ is a Poincaré duality algebra.

2. There exists an element $\omega$ in $H^{2}$ such that $\omega^{r}: H^{n-r} \rightarrow H^{n+r}$ is a vector space isomorphism for all $1 \leq r \leq n$.

Such an element $\omega$ is called a Kähler class for $H$.

Remark. The motivation for this definition comes from the fact that compact Kähler manifolds have an interesting cohomology structure, described by the hard Lefschetz theorem [W, V.4.13]. In the context of this paper, this theorem can be taken to read: Let $X$ be a compact Kähler manifold of real dimension $2 n$. Then there exists a distinguished class $\omega$ in $H^{2}$ such that the map $\omega^{r}: H^{n-r} \rightarrow H^{n+r}$, from taking the cup-product with $\omega r$-times, is an isomorphism for $1 \leq r \leq n$.

Spaces having such rational cohomology algebras have been considered in [Bor] and [Me]. Such spaces are referred to there as "homologically Kählerian." In [Bor, p. 170], results are given concerning fixed point sets relevant to such spaces. In [Me], the set of homotopy self-equivalences of such spaces is considered.

3.2. Examples. 1. By the above remark, there is a core of natural examples of Lefschetz algebras arising as the rational cohomology rings of Kähler manifolds. From the (rational homotopy) point of view of this paper, it is desirable to restrict attention to simply connected compact Kähler manifolds, although the hard Lefschetz theorem holds in the nonsimply connected case also. Examples of this kind include the rational cohomology of complex projective spaces $H^{*}\left(\mathbb{C} P^{n} ; \mathbb{Q}\right)$, the rational cohomology of complex Grassmannian manifolds $H^{*}(G(k, n) ; \mathbb{Q})$, and the rational cohomology of generalised flag manifoldssee also $\S 7$.

2. Examples of a more purely algebraic character can be constructed by considering graded algebras of the type $H=\Lambda(\omega, a) /\left(a^{2}-\omega^{k}, \omega^{n-k+1} a\right)$, with $|\omega|=2,|a|=2 k$ and $k$ satisfying $2 \leq 2 k \leq n$. It is easy to check that this is a Lefschetz algebra; and with care, simple examples such as this can be built up into quite complex Lefschetz algebras. 
3. It is well known that the cohomology of Kähler manifolds admits a Hodge decomposition [W, V.5.1], and this implies that the Betti numbers $b_{i}(X)$, for a compact Kähler manifold $X$, must be even for $i$ odd. Now, it is easy to check that $H$, given by

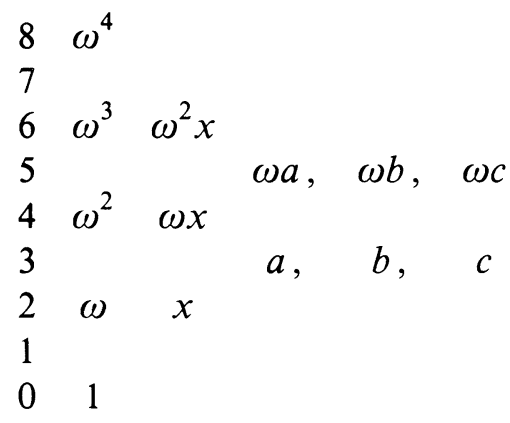

with $x^{2}=\omega^{2}, \omega^{3} x=0, a b=\omega^{3}+\omega^{2} x, a c=\omega^{3}-\omega^{2} x, b c=-\omega^{3}+\omega^{2} x$, $\omega^{2} a=\omega^{2} b=\omega^{2} c=0, c x=\omega a-\omega b, b x=\omega a+\omega c$, and $a x=\omega b-\omega c$, is a Poincare duality algebra, and so is clearly also a Lefschetz algebra. However, $\operatorname{rk}\left(H^{3}\right)=3$, so $H$ cannot be the cohomology algebra of any Kähler manifold. Together with 3.6-3.9 below, examples such as this give many Lefschetz algebras that do not come from Kähler manifolds.

4. Some intersection cohomology algebras provide examples of Lefschetz algebras [Mac].

The results of 3.6-3.9 below combined with the basic examples of 1 and 2 above make it possible to construct a large number of examples of Lefschetz algebras.

If $H$ is a Lefschetz algebra, there is a way of decomposing $H$-a kind of block decomposition-that is useful for displaying $H$, as a vector space, in such a way that it can help calculations.

3.3. Construction. Let $H$ be a Lefschetz algebra of dimension $2 n$ and consider the isomorphisms $\omega^{r}: H^{n-r} \rightarrow H^{n+r}$ for the various $r$ 's. Since $H^{0} \cong \mathbb{Q}, \omega^{n}$ is a nonzero multiple of the fundamental class of $H$ and none of the elements $\omega^{i}$ for $1<i<n$ can be zero either. Now, $\omega^{n-2}: H^{2} \rightarrow H^{2 n-2}$ is an isomorphism, but consider $\omega^{n-1}: H^{2} \rightarrow H^{2 n}$. Denote the kernel of this map by $V_{2}$; then $\omega$ is not in $V_{2}$. Furthermore,

$$
H^{2} \cong\langle\omega\rangle \oplus V_{2}
$$

and $\omega^{i}\left(H^{2}\right)$ is of rank equal to $\operatorname{rank}\left(H^{2}\right)$ for $1 \leq i \leq n-2$. Continuing in this fashion, increasing the degree by one at each stage, results in a vector space 
decomposition of $H$ which can be pictured as:

$\begin{array}{ccccccc}2 n & \omega^{n} & & & & \\ 2 n-1 & & & & & \\ 2 n-2 & \omega^{n-1} & \omega^{n-2} V_{2} & & & \\ 2 n-3 & & & \omega^{n-3} V_{3} & & \\ 2 n-4 & \omega^{n-2} & \omega^{n-3} V_{2} & & \omega^{n-4} V_{4} & \\ \vdots & \vdots & \vdots & \vdots & \vdots & \\ n & \cdots & \cdots & \cdots & \cdots & V_{n} \\ \vdots & \vdots & \vdots & \vdots & \vdots & \\ 4 & \omega^{2} & \omega V_{2} & & V_{4} & \\ 3 & & & V_{3} & & \\ 2 & \omega & V_{2} & & & \\ 1 & & & & & \\ 0 & 1 & & & & \end{array}$

where each $V_{j}=\operatorname{ker} \omega^{n-j+1}: H^{j} \rightarrow H^{2 n-j+2}$. Such a decomposition is known as a Lefschetz decomposition of $H$. Notice that all indecomposable elements of $H$-as an algebra-must belong to one of the spaces $V_{j}$; in particular, there can be no indecomposable elements of $H$ in degrees greater than $n$. Of course, apart from multiplication by $\omega$, such a decomposition does not say much about the multiplicative structure of $H$.

In the latter part of the paper, it will be desirable to choose a vector space basis for a Lefschetz algebra which takes into account both the Poincare duality structure and the Lefschetz structure. A construction is now given which can be used for this choice.

3.4. Construction. Assume $H$ has been decomposed as above; first, let the elements $\left\{\omega^{i}\right\}$ be a basis for the left-hand column. Now choose and fix bases for all the spaces $V_{j}$, say $V_{j}=\left\langle v_{j p}\right\rangle$. Consider the Poincare duals of elements in the vector space $V_{j}$, say, for $j<n$; these must all lie in $H^{2 n-j}$, which is spanned by $\left\{\omega^{n-j} V_{j}, \omega^{n-j+1} V_{j-2}, \ldots, \omega^{n-1-(j / 2)} V_{2}, \omega^{n-(j / 2)}\right\}$ if $j$ is even, and if $j$ is odd then the last two terms in this bracket must be replaced by $\omega^{n-(3 / 2)-(j / 2)} V_{3}$. But $\omega^{n-j+1}$ annihilates $V_{j}$ for $i \geq 1$, by construction, and so Poincaré duality must in fact induce nondegenerate bilinear pairings

$$
m: V_{j} \times \omega^{n-j} V_{j} \rightarrow \mathbb{Q}
$$

for ail $2 \leq j \leq n-1$. So let $\omega^{n-j} V_{j}=\left\langle v_{j p}^{*}\right\rangle$ be the dual bases to those chosen for the $V_{j}$ 's. Next, still for $j<n$, consider the epimorphism

$$
\omega^{t}: H^{2 n-j-2 t} \rightarrow H^{2 n-j},
$$

for all $t \geq 1$ such that $2 n-j-2 t \geq n+1$. This restricts to an isomorphism of vector spaces

$$
\omega^{t}: \omega^{n-j-t} V_{j} \rightarrow \omega^{n-j} V_{j} .
$$


Thus it is possible to choose bases $\omega^{n-j-t} V_{j}=\left\langle\left(\omega^{i}\right)^{-1} v_{j p}^{*}\right\rangle$. Finally, for all $i$ such that $\omega^{i} V_{j}$ is of degree less than or equal to $n$, fix bases as $\omega^{i} V_{j}=\left\langle\omega^{i} v_{j p}\right\rangle$, and $V_{n}=\left\langle v_{n p}\right\rangle$. This completes the construction.

Remark. By construction, $v_{j p} v_{j q}^{*}=\delta_{p q} \omega^{n}$. It is possible, without ambiguity, to denote the elements $\left(\omega^{t}\right)^{-1} v_{j p}^{*}$ by $\omega^{t} v_{j p}^{*}$, and these elements satisfy

$$
\omega^{t} v_{j p} \omega^{t} v_{j q}^{*}=v_{j p} \omega^{t}\left(\omega^{t}\right)^{-1} v_{j q}^{*}=\delta_{p q} \omega^{n}
$$

for all $j<n$. This notation will be adopted whenever a basis like this is used.

3.5. Notation. Let $H$ be a Lefschetz algebra. A vector space basis for $H$ will be called a Lefschetz-Poincaré basis for $H$ if it is chosen according to the above construction.

3.6. Proposition. Let $H$ and $G$ be Lefschetz algebras of dimension $2 n$ and $2 m$ and with Kähler classes $\omega$ and $x$ respectively. Then $H \otimes G$ is a Lefschetz algebra of dimension $2(n+m)$ and $\omega+x$ is a Kähler class for $H \otimes G$.

Proof. Since $H$ and $G$ are both Poincaré duality algebras, $H \otimes G$ is also a Poincaré duality algebra. It remains to check the Lefschetz property for $H \otimes G$, i.e., to check that

$$
(\omega+x)^{r}:(H \otimes G)^{n+m-r} \rightarrow(H \otimes G)^{n+m+r}
$$

is an isomorphism for $1 \leq r \leq n+m$. This can be done by first introducing the vector spaces $N_{k ; i, j}=\bigoplus_{p, q} \omega^{p} V_{i} \otimes x^{q} U_{j}$, where the sum is taken over those $p$ and $q$ for which $0 \leq p \leq n-i, 0 \leq q \leq m-j$, and $2 p+i+2 q+j=k$, i.e., $N_{k ; i, j} \subset(H \otimes G)^{k}$. Then it is clearly sufficient to check that

$$
(\omega+x)^{r}: N_{n+m-r ; i, j} \rightarrow N_{n+m+r ; i, j}
$$

is an isomorphism for each fixed pair $(i, j)$. To this end, for a given $r$, let $n+m-r-i-j=2 s$; i.e., let $2 s=2 p+2 q$ in the summands for $N_{n+m-r ; i, j}$. Then $N_{n+m-r ; i, j}=\bigoplus_{p+q=s} \omega^{p} V_{i} \otimes x^{q} U_{j}$, and it is sufficient to check that each

$$
(\omega+x)^{r}: \bigoplus_{p+q=s} \omega^{p} V_{i} \otimes x^{q} U_{j} \rightarrow \bigoplus_{p+q=r+s} \omega^{p} V_{i} \otimes x^{q} U_{j}
$$

is an isomorphism. Now each $\omega^{p} V_{i} \otimes x^{q} U_{j}$ is either zero, or isomorphic, as a vector space, to $V_{i} \otimes U_{j}$; so $(\omega+x)^{r}$ can be thought of as a linear transformation of column vectors

$$
(\omega+x)^{r}:(s+1) \text {-tuples } \rightarrow(r+s+1) \text {-tuples }
$$


and the corresponding matrix for $(\omega+x)^{r}$ is then the $r+s+1$ by $s+1$ matrix

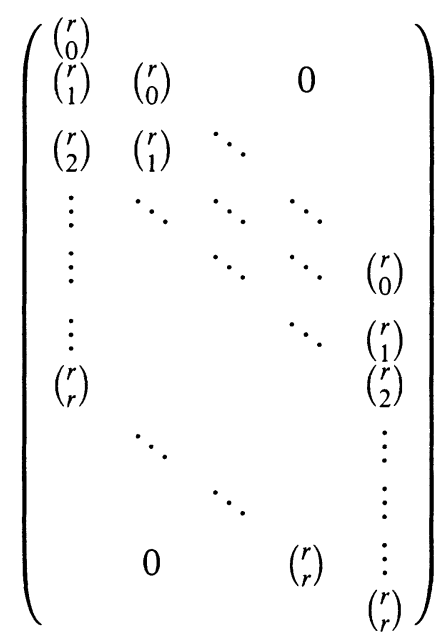

The remaining problem is to see just how, according as the value of $r$-that is, the value of $s$-changes, so the summands in the expressions for $N_{n+m-r ; i, j}$ and $N_{n+m+r ; i, j}$ become zero, at the same time as the nontrivial part of the above matrix changes. However, by splitting the possible values of $s$ into the three cases (1) $s \geq m-j$, (2) $s \geq n-j$, (3) $s<m-j$ and $s<n-i$, and then writing down the corresponding nontrivial part of the above matrix, it becomes an exercise in determinants to show that each case gives a nonsingular transformation of vector spaces-see my thesis [Lu] for details. Hence, $(\omega+x)^{r}: N_{n+m-r ; i, j} \rightarrow N_{n+m+r ; i, j}$ is an isomorphism, for each $r$ and a fixed $i$ and $j$. So, as remarked above, this implies

$$
(\omega+x)^{r}:(H \otimes G)^{n+m-r} \rightarrow(H \otimes G)^{n+m+r}
$$

is an isomorphism for each $1 \leq r \leq n+m$. Q.E.D.

3.7. Definition. Let $H$ and $G$ be Poincaré duality algebras of the same dimension with fundamental classes $\mu$ and $\nu$ respectively. The connected sum of $H$ and $G$ is the algebra $H \# G \cong(H \oplus G) /(\mu-\nu)$.

3.8. Proposition. Let $H$ be a Lefschetz algebra of dimension $2 n$ with Kähler class $\omega$. Let $G$ be an $(n-1)$-connected Poincaré duality algebra of dimension $2 n$. Then $H \# G$ is a Lefschetz algebra of dimension $2 n$, and $\omega$ is a Kähler class for $H \# G$.

Proof. $H \# G$ is Poincare duality, since this is so for any Poincaré duality algebras $H$ and $G$. The rest is clear. Q.E.D.

3.9. Proposition. Let $H$ and $G$ be Lefschetz algebras of dimension $2 n$. Then $H \# G$ is a Lefschetz algebra of dimension $2 n$.

Proof. It is sufficient to choose a Kähler class $\chi$ for $H \# G$, such that

$$
\chi^{r}:(H \# G)^{n-r} \rightarrow(H \# G)^{n+r}
$$


is an isomorphism for all $r$. If $\omega$ and $x$ are any choice of Kähler classes for $H$ and $G$ respectively, then for $1 \leq r<n, \chi=\omega+x$ gives maps $\chi^{r}=$ $\omega^{r}+x^{r}$, which split as the sum of two vector space isomorphisms, and hence are isomorphisms. Thus it is sufficient to choose Kähler classes $\omega$ and $x$ such that $(\omega+x)^{n}$ is nonzero in $H \# G$. If $\omega$ and $x$ are choices of Kähler classes, then so are $a \omega$ and $b x$, for $a$ and $b$ in $\mathbb{Q} \backslash\{0\}$. Say $\mu$ and $\nu$ are fundamental classes of $H$ and $G$, and that $\omega^{n}=\lambda_{1} \mu$ and $x^{n}=\lambda_{2} \nu$, for $\lambda_{1}$ and $\lambda_{2}$ nonzero. Then in $H \# G,(a \omega+b x)^{n}=a^{n} \omega^{n}+b^{n} x^{n}=a^{n} \lambda_{1} \mu+b^{n} \lambda_{2} \nu=\left(a^{n} \lambda_{1}+b^{n} \lambda_{2}\right) \mu$. Thus, it is sufficient to choose any Kähler classes $\omega$ and $x$, and then some pair $a$ and $b$ for which $\left(a^{n} \lambda_{1}+b^{n} \lambda_{2}\right)$ is nonzero; then $\chi=(a \omega+b x)$ is a Kähler class for $H \# G$. Q.E.D.

Finally, a result is proved that gives a kind of Poincare duality for Lefschetz algebras to do with elements multiplying to classes other than the fundamental class. This lemma will be used in the proof of Theorem 2 .

3.10. Notation. Denote a Lefschetz algebra $H(n, k)$ if it is of dimension $2 n$ and, apart from scalar multiples of powers of the Kähler class, is $(k-1)$ connected.

3.11. Lemma. Let $H=H(n, k)$ be a Lefschetz algebra, and $r$ be such that $\left|\omega^{r}\right|=2 r>2 n-k$; i.e., $\omega^{r}$ is in the range where $\operatorname{rank}\left(H^{i}\right)=0$ or 1 , above the middle degree. Then for any $x$ such that $|x| \leq r$, there exists some $\bar{x}$ such that $x \bar{x}=\omega^{r}$.

Proof. When $r=n$, Poincare duality gives the desired result. So consider $r$ in the range $2 n-k<2 r<2 n$, and let $x$ be some element of degree $\leq r$. Let $j$ be such that $x \in H^{r-j}$; then by Poincare duality there is an $x^{*}$ such that $x x^{*}=\omega^{n}$ and $\left|x^{*}\right|=2 n-(r-j)=n+(n-(r-j))$. But the isomorphism

$$
\omega^{n-(r-j)}: H^{n-(n-(r-j))} \rightarrow H^{n+(n-(r-j))}
$$

implies that $x^{*}=\omega^{n-(r-j)} \tilde{x}$ for some $\tilde{x}$. So $x \omega^{n-r} \omega^{j} \tilde{x}=\omega^{n}=\omega^{n-r} \omega^{r}$. Hence, $\left|x \omega^{j} \tilde{x}\right|=\left|\omega^{r}\right|$; and since $\operatorname{rk}\left(H^{2 r}\right)=1$, then $x \omega^{j} \tilde{x}=\lambda \omega^{r}$, for some scalar $\lambda$. But then $\omega^{n}=\omega^{n-r} x \omega^{j} \tilde{x}=\lambda \omega^{n}$, so $\lambda=1$. Now take $\bar{x}=$ $\omega^{j} \tilde{x}$. Q.E.D.

\section{DG LIE ALGEBRAS AND INTRINSIC FORMALITY}

In this section, attention is turned to the general intrinsic formality problem. Some results of a general nature are proved here, which are used in $\S \S 5$ and 6 in order to prove intrinsic formality results.

Several general results are known, concerning intrinsic formality; these have been proved using both DG algebras and DG Lie algebras. The result that lies at the heart of the strategy adopted in this paper, in order to prove intrinsic formality results, is a result using DG Lie algebras. 
4.1. Definition (cf. [N-Mi]). Let $\mathbf{L}(V, \partial)$ be a free $D G$ Lie algebra with a quadratic differential. Then $\mathbf{L}(V, \partial+P)$ is called a perturbation of $\mathbf{L}(V, \partial)$ if $\partial+P$ is a differential, and $P$ extends bracket lengths by at least two.

4.2. Theorem $\left[\mathrm{Mi}_{2}\right]$. Let $H$ be a 1-connected graded algebra. There exists a bijection of sets:

$$
\left\{\begin{array}{c}
\text { Rational homotopy } \\
\text { types having } \\
\text { cohomology } \cong H
\end{array}\right\} \leftrightarrow\left\{\begin{array}{c}
\text { Isomorphism classes } \\
\text { of perturbations } \\
\mathbf{L}\left(s^{-1} H_{*}, \partial_{\Delta}+P\right)
\end{array}\right\}
$$

4.3. Corollary. If $H$ is a graded algebra, and all perturbations of $\mathbf{L}\left(s^{-1} H_{*}, \partial_{\Delta}\right)$ are isomorphic to $\mathbf{L}\left(s^{-1} H_{*}, \partial_{\Delta}\right)$, then $H$ is intrinsically formal.

This corollary provides a strategy for considering the intrinsic formality problem, which will be adopted in this paper to prove intrinsic formality in certain cases. The same strategy has been used before to tackle, successfully, the intrinsic formality problem in other cases (see $\left[\mathrm{N}-\mathrm{Mi}, \mathrm{Mi}_{2}\right]$ ). In order to make use of this result in the next two sections, two results of a technical nature must be proved. The first of these is a rewording of a result in [St].

4.4. Proposition. Let $V$ be a 0-connected, graded vector space, and $\mathbf{L}(V, \partial)$ a free $D G$ Lie algebra with quadratic differential. Let $\mathbf{L}(V, \partial+P)$ be any perturbation and say

$$
\Phi: V \rightarrow \mathbf{L}(V)
$$

is a map of vector spaces of the form $\Phi=1+\psi$, where $\psi$ extends bracket lengths by at least one, but not necessarily homogeneously. Then $\Phi$ induces an isomorphic perturbation $\mathbf{L}(V, \partial+Q)$, which satisfies

$$
Q=P+\psi \partial+\psi P-\partial \psi-Q \psi
$$

Moreover, $\Phi$ is an isomorphism between these two perturbations.

Proof. $\Phi$ induces an obvious Lie algebra map $\Phi: \mathbf{L}(V) \rightarrow \mathbf{L}(V)$. Since $\Phi$ on $V$ is of the form $\Phi=1+\psi$, then $\Phi$ is in fact an isomorphism of Lie algebras. Write $\Phi=1+\psi: \mathbf{L}(V) \rightarrow \mathbf{L}(V)$. Now define $Q$ on $\mathbf{L}(V)$ as

$$
Q=(P+\psi \partial+\psi P-\partial \psi) \Phi^{-1} .
$$

Clearly $Q$ is well defined. It is now sufficient to check that $(\partial+Q)$ is a differential, and that $\Phi$ is a map of DG Lie algebras. By definition, for any $X$ in $\mathbf{L}(V)$,

$$
\begin{aligned}
Q \Phi(X) & =(P+\psi \partial+\psi P-\partial \psi) \Phi^{-1} \Phi(X) \\
& =P(X)+\psi \partial(X)+\psi P(X)-\partial \psi(X),
\end{aligned}
$$

and so

$$
(Q \Phi(X)+\partial \psi(X))=(P+\psi P+\psi \partial)(X)=(\Phi P+\psi \partial)(X)
$$


Adding $\partial X$ to both sides gives that $(\partial+Q) \Phi(X)=\Phi(\partial+P)(X)$. Now the commutative diagram

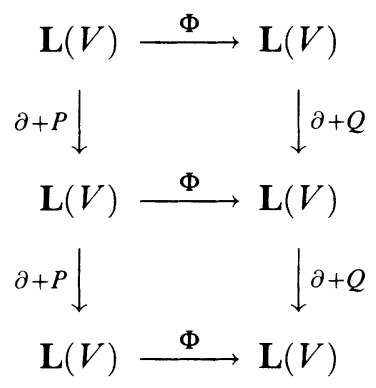

implies that $(\partial+Q)^{2}=0$, since $(\partial+P)^{2}=0$. Hence $(\partial+Q)$ is a differential, and so $\Phi$ is an isomorphism of DG Lie algebras. Q.E.D.

4.5. Notation. Let $\mathbf{L}(V, \partial)$ be a free $D G$ Lie algebra with quadratic differential. Then the bracket length of cycles representing classes of $H(\mathbf{L}(V, \partial))$ induces a filtration of $H(\mathbf{L}(V, \partial))$. If a class has a representative cycle of homogeneous bracket length, this length will be referred to as the underlying bracket length of the class. Notice that this is not necessarily the bracket length of the class in the Lie algebra $H(\mathbf{L}(V, \partial))$.

4.6. Proposition. Let $\mathbf{L}(V, \partial)$ be a free $D G$ Lie algebra with quadratic differential, and let $\mathbf{L}(V, \partial+P)$ be any perturbation. Assume the terms in $H(\mathbf{L}(V, \partial))$, of underlying bracket length greater than or equal to three, are zero in degrees $\leq r$. Then $\mathbf{L}(V, \partial+P)$ is isomorphic to a perturbation $\mathbf{L}(V, \partial+Q)$, where $Q$ is zero on elements of $\mathbf{L}(V)$ of degree $\leq r+1$.

Proof. A vector space map $(1+\psi): V \rightarrow \mathbf{L}(V)$ will be constructed that will allow the above proposition to be invoked. Construct $\psi$ inductively over degree as follows. On elements of degree 1, put $\psi$ equal to zero. Now assume $\psi$ has been constructed on elements of degree $\leq k \leq r$, such that, when $\Phi=1+\psi$ is extended to a Lie algebra map,

$$
\Phi(\partial+P)=\partial \Phi
$$

on all elements of $\mathbf{L}(V)$ of degree $\leq k$. Choose a basis for $V$ in degree $k+1$ and consider a basis element $v$. Then $(\partial+P)(v)$ is of degree $k$, so

$$
\partial \Phi(\partial+P)(v)=\Phi(\partial+P)^{2}(v)=0 .
$$

Thus $\partial(P+\psi \partial+\psi P)(v)=0$, and by the homology assumption, there exists some $\eta$ such that $(P+\psi \partial+\psi P)(v)=\partial \eta$. Furthermore, $\eta$ can be chosen to be of bracket length $\geq 2$. Put $\psi(v)=\eta$. This constructs a well-defined $\psi$ on $V$ in degree $k+1$. Now define $\Phi=1+\psi$ on elements of $V$ of degree $k+1$, and for such an element, by construction, $\Phi P(v)+\psi \partial(v)=\partial \psi(v)$, and hence $\Phi(\partial+P)=\partial \Phi$ on elements of $V$ in degree $k+1$. It is easy to check that if $(\dagger)$ holds on elements of $\mathbf{L}(V)$ in degrees $\leq k$, then it holds on brackets of such elements. Hence, $(\dagger)$ is true on all elements of $\mathbf{L}(V)$ in degrees $\leq k+1$, 
and induction goes through. This constructs $\Phi=1+\psi$ on all elements of $V$ in degrees $\leq r+1$. Define $\psi$ to be zero on any remaining elements of $V$. This completes the construction of $(1+\psi): V \rightarrow \mathbf{L}(V)$. Now invoke the above proposition, to induce an isomorphic perturbation $\mathbf{L}(V, \partial+Q)$, for which $Q=\Phi P+\psi \partial-\partial \psi-Q \psi$. On elements of $\mathbf{L}(V)$ of degree 1 , $Q$ is zero. Now inductively assume $Q$ to be zero on elements of $\mathbf{L}(V)$ of degrees $\leq k \leq r$, and let $X$ be an element of $\mathbf{L}(V)$ of degree $k+1$. Then $Q(X)=\Phi P(X)+\psi \partial(X)-\partial \psi(X)=0$, by $(\dagger)$. Therefore, by induction, $Q$ is zero on elements of $\mathbf{L}(V)$ of degrees $\leq r+1$. Q.E.D.

Finally, a technical result is given that provides linear independence criteria, useful for checking certain relations in free Lie algebras. This will be used in the next two sections.

4.7. Lemma. Let $V$ be a finite graded vector space and let $\left\{v_{1}, \ldots, v_{m}\right\}$ be a totally ordered basis of $V$. Let $\mathbf{L}(V)$ be the free Lie algebra on $V$, and $x$ in $\mathbf{L}(V)$ such that $x=\sum_{i}\left[v_{i}, X_{i}\right]$, where each $X_{i}$ is of the form

$$
X_{i}=\sum_{j} \lambda_{i}^{j}\left[v_{j_{1}},\left[v_{j_{2}}, \ldots,\left[v_{j_{r_{j}-1}}, v_{j_{r_{j}}}\right] \ldots\right]\right] \text {; }
$$

and for each bracket of $x$, of length $r_{j}+1, \lambda_{i}^{j}=0$ unless $\max \left\{v_{i}, v_{j_{1}}, \ldots, v_{j_{r_{j}-2}}\right\}$ $<v_{j_{r_{j}}}$, and $v_{j_{r_{j}-1}} \leq v_{j_{r_{j}}}$, with respect to the given order of the basis of $V$. If $x$ is zero in $\mathbf{L}(V)$, then each $X_{i}$ is equal to zero.

Proof. Straightforward, by considering the universal enveloping algebra of $\mathrm{L}(V)$, which is $T(V)$-the tensor algebra on $V[\mathrm{Q}$, appendix B]-and then using the fact that $\mathbf{L}(V)$ includes into $T(V)$, so the appropriate relations can be checked there. See my thesis [Lu] for details. Q.E.D.

\section{AN INTRINSIC FORMALITY RESULT}

This paper is primarily concerned with beginning the analysis of Lefschetz algebras from a rational homotopy point of view. However, it turns out that many of the simple cases of Lefschetz algebras to be considered in $\$ 7$ can in fact be treated as particular examples of a different type of algebra, which is not necessarily Lefschetz. Therefore, this section considers this different type of algebra and in particular investigates its intrinsic formality properties.

It is necessary to introduce some notation before stating the main result.

5.1. Definition. Let $H$ be a graded algebra. $H$ is an elliptic graded complete intersection if $H$ is a finite algebra and $H \cong \Lambda\left(x_{1}, \ldots, x_{n}\right) /\left(R_{1}, \ldots, R_{m}\right)$, where $\left\{R_{i}\right\}$ is a graded regular sequence (see [Józ]).

Remark. Such algebras are also called hyperformal in [Fé-H], where they need not be finite. These algebras have been well studied from the rational homotopy point of view $\left[\mathrm{H}_{2}, \mathrm{Fr}-\mathrm{H}\right]$. In particular, an elliptic graded complete intersection 
is intrinsically formal and is a Poincaré duality algebra $\left[\mathrm{H}_{2}\right]$. Furthermore, a minimal model for an $H$ as in the definition is given by

$$
\rho: \Lambda\left(x_{1}, \ldots, x_{n}, y_{1}, \ldots, y_{m} ; d\right) \rightarrow H,
$$

with $d x_{i}=0, d y_{j}=R_{j}$, and an obvious map $\rho$. Thus the rational homotopy of such an $H$ is finite, making the minimal model an elliptic space in the terminology of [Fé-H-Th]. Hence the notation introduced here.

Now the main result can be stated as

5.2. Theorem 1. Let $H \cong G_{1} \# G_{2}$, with $G_{1}$ an elliptic graded complete intersection of dimension $2 n$, and $G_{2}$ an $(n-1)$-connected Poincaré duality algebra of dimension $2 n$. Then $H$ is intrinsically formal.

Before proving the result, it is necessary to set up a number of technical lemmas. Recall the notation of 4.5 .

5.3. Lemma. Let $\mathbf{L}\left(s^{-1} H_{*}, \partial_{\Delta}\right)$ be the $D G$ Lie algebra minimal model of an elliptic graded complete intersection $H$. Then the only nonzero terms in $H\left(\mathbf{L}\left(s^{-1} H_{*}, \partial_{\Delta}\right)\right)$ are sums of classes of underlying bracket length 1 and 2 .

Proof. By the well-known correspondence between elements in $Z_{0}$ and $Z_{1}$ of the bigraded model of $H$, and underlying bracket length 1 and 2 terms of $H\left(\mathbf{L}\left(s^{-1} H_{*}, \partial_{\Delta}\right)\right)$. Q.E.D.

5.4. Proposition. Let $H \cong G_{1} \# G_{2}$, where $G_{1}$ is an elliptic graded complete intersection of dimension $2 n$, and $G_{2}$ is an $(n-1)$-connected Poincaré duality algebra of dimension $2 n$. Let $\mathbf{L}\left(s^{-1} H_{*}, \partial_{\Delta}\right)$ be the $D G$ Lie algebra minimal model of $H$, and let $W$ be the Lie ideal in $\mathrm{L}\left(s^{-1} H_{*}\right)$ generated by $s^{-1} G_{2}^{n}$. Then the underlying bracket length $\geq 3$ terms of $H\left(\mathbf{L}\left(s^{-1} H_{*}, \partial_{\Delta}\right)\right)$ are spanned by classes with representatives in $W$.

Proof. Consider the short exact sequence

$$
\begin{aligned}
0 & \rightarrow \mathbf{L}\left(s^{-1} G_{2 *}^{n}, A D\left(s^{-1} G_{1 *}\right)\left(s^{-1} G_{2 *}^{n}\right) ; d\right) \\
& \stackrel{j}{\rightarrow} \mathbf{L}\left(s^{-1} H_{*}, \partial_{\Delta}\right) \stackrel{p}{\rightarrow} \mathbf{L}\left(s^{-1} G_{1 *}, \partial_{\Delta}\right) \rightarrow 0 .
\end{aligned}
$$

This induces a long exact sequence in homology. Since the two right-hand terms have quadratic differentials, $p_{*}$ preserves underlying bracket lengths, and since $G_{1}$ is an elliptic complete intersection, $H\left(\mathbf{L}\left(s^{-1} G_{1 *}, \partial_{\Delta}\right)\right)$ has no terms of underlying bracket length $\geq 3$. Thus, for all underlying bracket length $\geq 3$ terms in $H\left(\mathbf{L}\left(s^{-1} H_{*}, \partial_{\Delta}\right)\right), j_{*}$ is surjective. Q.E.D.

5.5. Corollary. Let $H \cong G_{1} \# G_{2}$ as above, and let $\mathbf{L}\left(s^{-1} H_{*}, \partial_{\Delta}+P\right)$ be a perturbation of $\mathbf{L}\left(s^{-1} H_{*}, \partial_{\Delta}\right)$. Then $\mathbf{L}\left(s^{-1} H_{*}, \partial_{\Delta}+P\right)$ is isomorphic to a perturbation $\mathbf{L}\left(s^{-1} H_{*}, \partial_{\Delta}+Q\right)$, where $Q=0$ on elements of $\mathbf{L}\left(s^{-1} H_{*}\right)$ of degree $\leq n+1$.

Proof. From above, all underlying bracket length $\geq 3$ terms in the homology $H\left(\mathbf{L}\left(s^{-1} H_{*}, \partial_{\Delta}\right)\right)$ are represented by elements of the ideal $W$. But $W$ is zero 
in degrees $\leq n-2$, so bracket length $\geq 3$ terms in $W$ are zero in degrees $\leq n$. Hence, underlying bracket length $\geq 3$ terms in $H\left(\mathbf{L}\left(s^{-1} H_{*}, \partial_{\Delta}\right)\right)$ are zero in degrees $\leq n$, and by 4.6 , the corollary holds. Q.E.D.

5.6. Proposition. Let $H \cong G_{1} \# G_{2}$ be as in the above proposition, let $W$ be the ideal in $\mathbf{L}\left(s^{-1} H_{*}\right)$ as above, and let $\mathbf{L}\left(s^{-1} H_{*}, \partial_{\Delta}+P\right)$ be a perturbation of $\mathbf{L}\left(s^{-1} H_{*}, \partial_{\Delta}\right)$. Then $\mathbf{L}\left(s^{-1} H_{*}, \partial_{\Delta}+P\right)$ is isomorphic to a perturbation $\mathbf{L}\left(s^{-1} H_{*}, \partial_{\Delta}+Q\right)$ for which $\operatorname{im} Q \subset W$.

Proof. Assume inductively that $\operatorname{im} P \subset W$ for $P$ acting on elements of $\mathbf{L}\left(s^{-1} H_{*}\right)$ of degrees $\leq r$, some $r \leq 2 n-2$. Construct a vector space map $\Phi=1+\psi: s^{-1} H_{*} \rightarrow \mathbf{L}\left(s^{-1} H_{*}\right)$ as follows. On elements of $s^{-1} H_{*}$ of degrees $\leq r$, put $\psi=0$. Choose a basis for $s^{-1} H_{*}$ in degree $r+1$, and let $v$ be a basis element. Now consider $P(v) . \partial_{\Delta}+P$ is a differential, so $\left(\partial_{\Delta} P+P P+P \partial_{\Delta}\right)(v)=0$, and hence $\partial_{\Delta} P(v) \subset W$, by the inductive assumption. In the short exact sequence of DG Lie algebras

$$
\begin{aligned}
0 & \rightarrow \mathbf{L}\left(s^{-1} G_{2 *}^{n}, A D\left(s^{-1} G_{1 *}\right)\left(s^{-1} G_{2 *}^{n}\right) ; d\right) \\
& \stackrel{j}{\rightarrow} \mathbf{L}\left(s^{-1} H_{*}, \partial_{\Delta}\right) \stackrel{p}{\rightarrow} \mathbf{L}\left(s^{-1} G_{1 *}, \partial_{\Delta}\right) \rightarrow 0 ;
\end{aligned}
$$

$\partial_{\Delta}(p P(v))=p\left(\partial_{\Delta} P(v)\right)=0$, since $\partial_{\Delta} P(v) \subset W=\operatorname{im}(j)$. Thus $p P(v)$ is a cycle in $\mathbf{L}\left(s^{-1} G_{1 *}, \partial_{\Delta}\right)$ of bracket length $\geq 3$, and hence is exact by 5.2. Say $p P(v)=\partial_{\Delta} \zeta$, for some $\zeta . p$ is surjective, so $\zeta=p(\eta)$, for some $\eta$, whence $p\left(P(v)-\partial_{\Delta} \eta\right)=0$, and so by exactness, $P(v)-\partial_{\Delta}(\eta)=j(\theta)$, some $\theta$. So, given a basis element $v$ in $s^{-1} H_{*}$ of degree $r+1$, as above, choose some $\eta$ and $\theta$ in $W$ such that

$$
P(v)=j(\theta)+\partial_{\Delta} \eta,
$$

and define $\psi(v)=\eta$. Thus $\psi$ is well defined in degrees $\leq r+1$. Now define $\psi$ to be zero in degrees $\geq r+2$. This completes the construction of $\Phi$. As in 4.4, this induces an isomorphic perturbation $\mathrm{L}\left(s^{-1} H_{*}, \partial_{\Delta}+Q\right)$, which satisfies

$$
Q=P+\psi \partial_{\Delta}+\psi P-\partial_{\Delta} \psi-Q \psi .
$$

On elements of $s^{-1} H_{*}$ of degree $\leq r$, and all brackets of such elements, $\psi$ is zero, and so $Q=P$ here. In degree $r+1, Q(v)=P(v)-\partial_{\Delta} \psi(v)-Q \psi(v)=$ $P(v)-\partial_{\Delta} \eta-Q \psi(v)=j(\theta)-P \psi(v) \subset W$ by construction, and hence $\operatorname{im} Q \subset W$ for $Q$ acting on elements of $\mathbf{L}\left(s^{-1} H_{*}\right)$ of degree $\leq r+1$. So induction goes through. By 5.5 it is possible to assume that $P=0$ on elements of degree $\leq n+1$. Thus, by induction, the proposition is proven. Q.E.D.

Having set up the technical preliminaries, it is now possible to prove the main theorem of this section.

5.7. Proof of Theorem 1. First, fix a basis for the DG Lie algebra minimal model of $H$ as follows. Choose a basis for $G_{1},\left\{1, x_{i}, \mu\right\}$ say. Then by Poincaré duality, there exists a dual basis $\left\{1, x_{i}^{*}, \mu\right\}$. For $G_{1}$ in degrees $<n$ choose 
the basis elements $\left\{1, x_{i}\right\}_{i \in I}$. For $G_{1}$ in degree $n$, choose basis elements $\left\{x_{j}\right\}_{j \in J}$; and for $G_{1}$ in degrees $>n$, choose the basis elements $\left\{x_{i}^{*}, \mu\right\}_{i \in I}$. So $\left\{1, x_{i}, x_{i}^{*}, x_{j}, \mu\right\}_{i \in I, j \in J}$ is a basis for $G_{1}$. For $G_{2}$ in degree $n$, choose any basis $\left\{y_{k}\right\}_{k \in K}$. Then $\left\{1, x_{i}, x_{i}^{*}, x_{j}, y_{k}, \mu\right\}_{i \in I, j \in J, k \in K}$ is a basis for $H$. By abuse of notation, retain the same letters for the dualised basis of the coalgebra $H_{*}$; then $\left\{s^{-1} x_{i}, s^{-1} x_{i}^{*}, s^{-1} x_{j}, s^{-1} y_{k}, s^{-1} \mu\right\}$ is the desired basis for $\mathbf{L}\left(s^{-1} H_{*}, \partial_{\Delta}\right)$.

Let $W$ be the ideal in $\mathbf{L}\left(s^{-1} H_{*}\right)$ generated by the $\left\{s^{-1} y_{k}\right\}$. Let the DG Lie algebra $\mathbf{L}\left(s^{-1} H_{*}, \partial_{\Delta}+P\right)$ be any perturbation of $\mathbf{L}\left(s^{-1} H_{*}, \partial_{\Delta}\right)$. Without loss of generality, by 5.5 and 5.6, assume that $P=0$ on elements of $\mathbf{L}\left(s^{-1} H_{*}\right)$ of degrees $\leq n+1$, and that $\operatorname{im} P \subset W$. From the construction of the basis of $s^{-1} H_{*}$,

$$
\begin{aligned}
\partial_{\Delta}\left(s^{-1} \mu\right)= & \sum_{i \in I}(-1)^{\left|s^{-1} x_{i}\right|}\left[s^{-1} x_{i}, s^{-1} x_{i}^{*}\right]+\sum_{a, b \in J} \lambda_{a, b}\left[s^{-1} x_{a}, s^{-1} x_{b}\right] \\
& +\sum_{c, d \in K} \nu_{c, d}\left[s^{-1} y_{c}, s^{-1} y_{d}\right] .
\end{aligned}
$$

Furthermore, it is possible to write, using the Jacobi identity,

$$
P\left(s^{-1} \mu\right)=\sum_{i \in I}\left[s^{-1} x_{i}, R_{i}\right]
$$

with each element $R_{i}$ in $W$, by assumption on $P$; and furthermore, every bracket of each $R_{i}$ has exactly one entry from $\left\{s^{-1} y_{k}\right\}$, for dimensional reasons, so each bracket of $R_{i}$ can, and will, be assumed to be written as a "nested" or "right justified" bracket $\left[s^{-1} x_{i_{1}},\left[\ldots,\left[s^{-1} x_{i_{r}}, s^{-1} y_{s}\right] \cdots\right]\right]$, up to a scalar, with the $s^{-1} y_{s}$ entry as the right-hand entry. Using $\left(\dagger_{1}\right)$ and $\left(\dagger_{2}\right)$, define a vector space map $\Phi=1+\psi: s^{-1} H_{*} \rightarrow \mathbf{L}\left(s^{-1} H_{*}\right)$ by putting $\psi\left(s^{-1} x_{i}^{*}\right)=-(-1)^{\left|s^{-1} x_{i}\right|} R_{i}$, and $\psi=0$ on all other basis elements. By 4.4 this defines an isomorphic perturbation $\mathbf{L}\left(s^{-1} H_{*}, \partial_{\Delta}+Q\right)$ such that, if $\Phi=1+\psi$ is a map of Lie algebras,

$$
Q=P+\psi \partial_{\Delta}-\partial_{\Delta} \psi+\psi P-Q \psi .
$$

Claim. $Q$ is zero.

Proof of claim. $P$ and $\psi$ are both zero on elements in $\left\{s^{-1} x_{i}, s^{-1} x_{j}, s^{-1} y_{k}\right\}$, and using $\left(\dagger_{3}\right), Q$ is zero on these elements also. This leaves the elements $\left\{x_{i}^{*}, \mu\right\}$. In a similar fashion to the argument in [St], consider the two equations

$$
0=\Phi \partial_{\Delta}^{2}\left(s^{-1} \mu\right)=\sum_{i \in I}(-1)^{\left|s^{-1} x_{i}\right|}\left[\partial_{\Delta} s^{-1} x_{i}, \psi\left(s^{-1} x_{i}^{*}\right)\right]+\sum_{i \in I}\left[s^{-1} x_{i}, \psi \partial_{\Delta}\left(s^{-1} x_{i}^{*}\right)\right]
$$


and

$$
\begin{aligned}
0= & \left(\partial_{\Delta}+P\right)^{2}\left(s^{-1} \mu\right)=\left(\partial_{\Delta} P+P P+P \partial_{\Delta}\right)\left(s^{-1} \mu\right) \\
= & \sum_{i \in I}\left[\partial_{\Delta} s^{-1} x_{i}, R_{i}\right]+\sum_{i \in I}(-1)^{\left|s^{-1} x_{i}\right|}\left[s^{-1} x_{i}, \partial_{\Delta} R_{i}\right] \\
& +\sum_{i \in I}\left[s^{-1} x_{i}, P\left(s^{-1} x_{i}^{*}\right)\right]+\sum_{i \in I}(-1)^{\left|s^{-1} x_{i}\right|}\left[s^{-1} x_{i}, P\left(R_{i}\right)\right] .
\end{aligned}
$$

By construction, adding these latter two equations gives

$$
0=\sum_{i \in I}\left[s^{-1} x_{i}, P\left(s^{-1} x_{i}^{*}\right)+\psi \partial_{\Delta}\left(s^{-1} x_{i}^{*}\right)-\partial_{\Delta} \psi\left(s^{-1} x_{i}^{*}\right)\right],
$$

since $P\left(R_{i}\right)$ is zero, from a consideration of the entries in each $R_{i}$. Now, $\operatorname{im} P \subset W$, and by construction $\operatorname{im} \psi \subset W$ also. $W$ is clearly stable under $\partial_{\Delta}$, and so after placing an obvious total order on the basis of $s^{-1} H_{*}$, whereby elements $\left\{s^{-1} y_{s}\right\}$ have maximal order, this equation fits into the hypotheses of 4.7. Thus, for each $i,\left(P+\psi \partial_{\Delta}-\partial_{\Delta} \psi\right)\left(s^{-1} x_{i}^{*}\right)=0$. A simple induction argument over degree, using $\left(\dagger_{3}\right)$ and the fact that $\psi P\left(s^{-1} x_{i}^{*}\right)=0$, implies that $Q=0$ on all elements $\left\{s^{-1} x_{i}^{*}\right\}$. This leaves $Q\left(s^{-1} \mu\right)=P\left(s^{-1} \mu\right)+\psi \partial_{\Delta}\left(s^{-1} \mu\right)+$ $\psi P\left(s^{-1} \mu\right)$ by $\left(\dagger_{3}\right)$. But $\psi P\left(s^{-1} \mu\right)=0$, and hence $Q\left(s^{-1} \mu\right)=P\left(s^{-1} \mu\right)+$ $\psi \partial_{\Delta}\left(s^{-1} \mu\right)=0$ by construction. This proves the claim.

Finally, since $\partial_{\Delta}+P$ was an arbitrary perturbation, the result of 4.3 implies $H$ is intrinsically formal. Q.E.D.

\section{A SECOND INTRINSIC FORMALITY RESULT}

In this section the second main result is proven-6.1. Unlike the main result of the previous section, this one applies only to Lefschetz algebras. For this section it will be assumed that any Lefschetz algebra already has a LefschetzPoincaré basis, chosen as in $\S 3$. Recall the notation of 3.10. The main result can be stated as

6.1. Theorem 2. Let $H=H(n, k)$ be a Lefschetz algebra. If $2 n \leq 4 k-2$, then $H$ is intrinsically formal. ${ }^{1}$

Remark. This is the Lefschetz algebra analogue of Miller's result $\left[\mathrm{Mi}_{2}\right]$.

Once again, before proving the result, it is necessary to set up a number of technical lemmas. In order to begin these, it is necessary to know something about the rational homotopy of a Lefschetz algebra. The appropriate information is provided by the following result, which is proved in my thesis [Lu, appendix]. Recall the notation of 3.4 and 4.5 .

6.2. Proposition. Let $H=H(n, k)$ be a Lefschetz algebra, with $2 n \leq 4 k-$ 2. Then in degrees $\leq 2 n-2$, the underlying bracket length $\geq 3$ terms of

${ }^{1} \mathrm{~S}$. Papadima kindly informed me in a letter that B. Berceanu of INCREST, Romania, has proved a result very similar to this. 
$H\left(\mathbf{L}\left(s^{-1} H_{*}, \partial_{\Delta}\right)\right)$ are spanned, as a vector space, by classes with the following representatives:

1. Underlying bracket length 3-terms $\left[s^{-1} v_{i j}, X_{i j}\right]$, where $\partial_{\Delta}\left(s^{-1} v_{i j}\right)=0$, $X_{i j}$ is $\partial_{\Delta}$-closed, of bracket length 2 , and of degree $\geq n-1$.

2. Underlying bracket length 4-terms $\left[s^{-1} v_{k p},\left[s^{-1} v_{k q},\left[s^{-1} v_{k r}, s^{-1} v_{k s}\right]\right]\right]$ in degree $2 n-2$ if $2 n=4 k-2$; and if $2 n<4 k-2$, all underlying bracket length $\geq 4$ terms of $H\left(\mathbf{L}\left(s^{-1} H_{*}, \partial_{\Delta}\right)\right)$ of degree $\leq 2 n-2$ are zero.

All underlying bracket length $\geq 5$ terms of degree $\leq 2 n-2$ are zero.

The proof of this result takes the form of a fairly lengthy, steady calculation, using the Lefschetz property of $H$ and the way in which this multiplicative structure is reflected in the differential of the Quillen model of $H$.

6.3. Corollary. Let $H=H(n, k)$ be a Lefschetz algebra with $2 n \leq 4 k-2$. If $\mathbf{L}\left(s^{-1} H_{*}, \partial_{\Delta}+P\right)$ is any perturbation of the Quillen model of $H$, then there exists an isomorphic perturbation $\mathbf{L}\left(s^{-1} H_{*}, \partial_{\Delta}+Q\right)$ such that $Q=0$ except on elements $s^{-1} \omega^{i}$ of degree $>n+k-2$.

Proof. By the above result, underlying bracket length $\geq 3$ homology of the model $\mathbf{L}\left(s^{-1} H_{*}, \partial_{\Delta}\right)$ is zero in degrees $\leq n+k-3$. Now apply 4.6. Q.E.D.

A substantial part of the theorem can now be proved. The idea behind the proof is as follows. The corollary above means that it is possible to assume a perturbation of the Quillen model of $H$ is only nonzero on elements $s^{-1} \omega^{i}$ of sufficiently high degree that they are in the range where rank $H^{r}=0$ or 1 , above the middle degree. Suppose the only element on which $P$ is nonzero is $s^{-1} \omega^{n}$. Then using the "Miller-Stasheff" techniques for removing perturbations off the fundamental classes of Poincaré duality algebras, it would be possible to show $H$ intrinsically formal directly. Here, however, the situation is more complicated, but is helped by the fact that, for the purposes of removing perturbations à la Miller-Stasheff, the $\omega^{i}$,s for $i<n$ behave like little fundamental classes for the relevant terms. This is made precise by 3.11 .

6.4. Notation. Denote by $P_{3}$ that part of a perturbation $\partial+P$ that extends bracket lengths by two (see [St]).

6.5. Proposition. Let $H=H(n, k)$ be a Lefschetz algebra with $2 n \leq 4 k-2$, and $\mathbf{L}\left(s^{-1} H_{*}, \partial_{\Delta}+P\right)$ be a perturbation of the Quillen model of $H$ such that $P=0$ on all elements of degree $\leq n+k-2$. Then there exists an isomorphic perturbation $\mathbf{L}\left(s^{-1} H_{*}, \partial_{\Delta}+Q\right)$ with $Q_{3}=0$.

Proof. Assume that the element of lowest degree on which $P_{3}$ is nonzero is $s^{-1} \omega^{p}$. A linear map $\psi: s^{-1} H_{*} \rightarrow \mathbf{L}\left(s^{-1} H_{*}\right)$ will be constructed so that, by 4.4, an isomorphic perturbation is induced by $\Phi=1+\psi: \mathbf{L}\left(s^{-1} H_{*}, \partial_{\Delta}+P\right) \rightarrow$ $\mathbf{L}\left(s^{-1} H_{*}, \partial_{\Delta}+Q\right)$ and $Q_{3}$ will be zero on elements of degree $\leq\left|s^{-1} \omega^{p}\right|$, and $Q=0$ on elements of degree $\leq n+k-2$. 
That $\left(\partial_{\Delta}+P\right)^{2}=0$ implies that $P_{3}\left(s^{-1} \omega^{p}\right)$ is a $\partial_{\Delta}$-cycle of bracket length 3. By 6.2 above, it can be written

$$
P_{3}\left(s^{-1} \omega^{p}\right)=\partial_{\Delta} \alpha+\sum\left[s^{-1} v_{i j}, X_{i j}\right]
$$

with $\left|X_{i j}\right| \geq n-1$ and $\left|v_{i j}\right|<\frac{1}{2}\left|\omega^{p}\right|$. Using the Lefschetz-Poincaré basis, it is also possible to write

(2)

$$
\begin{aligned}
\partial_{\Delta}\left(s^{-1} \omega^{p}\right)= & \sum\left[s^{-1} v_{i j}, s^{-1} \omega^{n-p} v_{i j}^{*}\right]+\sum\left[s^{-1} \omega v_{i j}, s^{-1} \omega^{n-p+1} v_{i j}^{*}\right] \\
& +\cdots+\sum\left[s^{-1} \omega^{q_{i}} v_{i j}, s^{-1} \omega^{n-p+q_{i}} v_{i j}^{*}\right]+\sum\left[s^{-1} \omega^{i}, s^{-1} \omega^{p-i}\right] \\
& +\sum \text { terms with entries of } \operatorname{deg} \leq n-1,
\end{aligned}
$$

where $q_{i}$ is the largest integer such that $\left|\omega^{n-p+q_{i}} v_{i j}\right|<n$. Furthermore, the indexing set for the sum in (1) is the same set as the indexing set for the first sum on the right-hand side of (2), by 3.11. From (1) and (2) the construction of $\psi$ can be started as: $\psi=0$ on all elements of degree $\leq n-1$, and on all elements $s^{-1} \omega^{n}$, for $r<p ; \psi\left(s^{-1} \omega^{p}\right)=\alpha, \psi\left(s^{-1} \omega^{n-p} v_{i j}^{*}\right)=-(-1)^{\left|v_{i j}\right|} X_{i j}$ and $\psi\left(s^{-1} \omega^{r} v_{i j}^{*}\right)=0$ for $\left|s^{-1} \omega^{r} v_{i j}^{*}\right|<\left|s^{-1} \omega^{n-p} v_{i j}^{*}\right|$.

To extend $\psi$ over the remaining elements of degree $>\left|s^{-1} \omega^{n-p} v_{i}^{*}\right|$, in the $s^{-1} V_{i}$ column-see the following table:

$$
\begin{array}{cccc}
2 n-i-1 & s^{-1} v_{i_{1}}^{*}, & \cdots, & s^{-1} v_{i_{\nu(i)}^{*}}^{*} \\
2 n-i-3 & s^{-1} \omega v_{i_{1}}^{*}, & \cdots, & s^{-1} \omega v_{i_{\nu(i)}^{*}}^{*} \\
\vdots & \vdots & & \vdots \\
2 p-i+1 & s^{-1} \omega^{n-p-1} v_{i_{1}}^{*}, & \cdots, & s^{-1} \omega^{n-p-1} v_{i_{\nu(i)}}^{*} \\
2 p-i-1 & s^{-1} \omega^{n-p} v_{i_{1}}^{*}, & \cdots, & s^{-1} \omega^{n-p} v_{i_{\nu(i)}^{*}}^{*} \\
2 p-i-3 & \psi_{2}=0 \text { on all elements } \leq \text { this degree in this column }
\end{array}
$$

and for each $i$, it is necessary to prove

Claim. On the elements $\left\{s^{-1} \omega^{n-p-t} v_{i j}^{*}\right\}_{t=0, \ldots, n-p}$, for each $i j, \psi$ can be defined inductively such that

$$
\begin{aligned}
\partial_{\Delta} \psi\left(s^{-1} \omega^{n-p-t} v_{i j}^{*}\right)= & -\left[s^{-1} \omega, \psi\left(s^{-1} \omega^{n-p-(t-1)} v_{i j}^{*}\right)\right] \\
& -\cdots-\left[s^{-1} \omega^{t}, \psi\left(s^{-1} \omega^{n-p} v_{i j}^{*}\right)\right]
\end{aligned}
$$

Proof of claim. For $t=0, \psi$ is already defined on $s^{-1} \omega^{n-p} v_{i j}^{*}$, as

$$
\psi\left(s^{-1} \omega^{n-p} v_{i j}^{*}\right)= \pm X_{i j}
$$

with $\partial_{\Delta} X_{i j}=0$. So assume that $\psi\left(s^{-1} \omega^{n-p-r} v_{i j}^{*}\right)$ is defined for all $r \leq t-1$ 
as in the hypothesis, and consider the formula for $\Phi \partial_{\Delta}\left(s^{-1} \omega^{n-p-t} v_{i j}^{*}\right)$.

$$
\begin{aligned}
(1+\psi) \partial_{\Delta}\left(s^{-1} \omega^{n-p-t} v_{i j}^{*}\right)= & \partial_{\Delta}\left(s^{-1} \omega^{n-p-t} v_{i j}^{*}\right)-\left[s^{-1} \omega, \psi s^{-1} \omega^{n-p-(t-1)} v_{i j}^{*}\right] \\
& -\left[s^{-1} \omega^{2}, \psi s^{-1} \omega^{n-p-(t-2)} v_{i j}^{*}\right] \\
& -\cdots-\left[s^{-1} \omega^{t}, \psi s^{-1} \omega^{n-p} v_{i j}^{*}\right] \\
& +\psi(\text { terms on which } \psi=0) .
\end{aligned}
$$

Applying $\partial_{\Delta}$ gives

$$
\begin{aligned}
\partial_{\Delta} \Phi \partial_{\Delta} & \left(s^{-1} \omega^{n-p-t} v_{i j}^{*}\right) \\
= & {\left[s^{-1} \omega, \partial_{\Delta} \psi\left(s^{-1} \omega^{n-p-(t-1)} v_{i j}^{*}\right)\right]+\left[s^{-1} \omega^{2}, \partial_{\Delta} \psi\left(s^{-1} \omega^{n-p-(t-2)} v_{i j}^{*}\right)\right] } \\
& +\cdots+\left[s^{-1} \omega^{t}, \partial_{\Delta} \psi\left(s^{-1} \omega^{n-p} v_{i j}^{*}\right)\right]-\left[\partial_{\Delta}\left(s^{-1} \omega\right), \psi\left(s^{-1} \omega^{n-p-(t-1)} v_{i j}^{*}\right)\right] \\
& -\left[\partial_{\Delta}\left(s^{-1} \omega^{2}\right), \psi\left(s^{-1} \omega^{n-p-(t-2)} v_{i j}^{*}\right)\right]-\cdots-\left[\partial_{\Delta}\left(s^{-1} \omega^{t}\right), \psi\left(s^{-1} \omega^{n-p} v_{i j}^{*}\right)\right] .
\end{aligned}
$$

The right-hand side can be expanded using the induction hypothesis for the first set of terms, and the fact that $\left|s^{-1} \omega^{t}\right|<2 k$, giving

$$
\partial_{\Delta}\left(s^{-1} \omega^{r}\right)=\sum-\left[s^{-1} \omega^{i}, s^{-1} \omega^{r-i}\right]
$$

for such $r$ in the second set of terms. This gives

$$
\left.\begin{array}{rl}
\partial_{\Delta} \psi & \partial_{\Delta}\left(s^{-1} \omega^{n-p-t} v_{i j}^{*}\right) \\
= & -\left[s^{-1} \omega,\left\{\left[s^{-1} \omega, \psi\left(s^{-1} \omega^{n-p-(t-2)} v_{i j}^{*}\right)\right]+\left[s^{-1} \omega^{2}, \psi\left(s^{-1} \omega^{n-p-(t-3)} v_{i j}^{*}\right)\right]\right.\right. \\
& \left.\left.+\cdots+\left[s^{-1} \omega^{t-1}, \psi\left(s^{-1} \omega^{n-p} v_{i j}^{*}\right)\right]\right\}\right]
\end{array}\right]
$$

where in this last line, $[t / 2]$ is the largest integer less than or equal to $t / 2$ and $\langle t / 2\rangle$ is the smallest integer greater than or equal to $t / 2$; and if these latter two are equal, then a $1 / 2$ should be placed before the bracket containing these terms. But for each triple $\left\{s^{-1} \omega^{b}, s^{-1} \omega^{c}, \psi\left(s^{-1} \omega^{n-p-a} v_{i j}^{*}\right)\right\}$, where $1 \leq t-1$, and $a+b+c=t$, it is clear that the above equation contains copies of the 
Jacobi identities:

for $b \neq c$

$$
\begin{aligned}
0= & -\left[s^{-1} \omega^{c},\left[s^{-1} \omega^{b}, \psi\left(s^{-1} \omega^{n-p-a} v_{i j}^{*}\right)\right]\right] \\
& -\left[s^{-1} \omega^{b},\left[s^{-1} \omega^{c}, \psi\left(s^{-1} \omega^{n-p-a} v_{i j}^{*}\right)\right]\right] \\
& +\left[\left[s^{-1} \omega^{c}, s^{-1} \omega^{b}\right], \psi\left(s^{-1} \omega^{n-p-a}\right)\right]
\end{aligned}
$$

and for $b=c$

$$
\begin{aligned}
0= & -\left[s^{-1} \omega^{b},\left[s^{-1} \omega^{b}, \psi\left(s^{-1} \omega^{n-p-a} v_{i j}^{*}\right)\right]\right] \\
& +\frac{1}{2}\left[\left[s^{-1} \omega^{b}, s^{-1} \omega^{b}\right], \psi\left(s^{-1} \omega^{n-p-a} v_{i j}^{*}\right)\right] .
\end{aligned}
$$

Notice that in these latter formulae, $s^{-1} \omega^{b}$ and $s^{-1} \omega^{c}$ have odd degree. Furthermore, these terms are all that the right-hand side of the equation contains. Thus $\partial_{\Delta} \psi \partial_{\Delta}\left(s^{-1} \omega^{n-p-t} v_{i j}^{*}\right)=0$, so by 6.2 above, $\psi \partial_{\Delta}\left(s^{-1} \omega^{n-p-t} v_{i j}^{*}\right)$ is exact, since it is a length $\geq 3$ cycle of degree $\leq n+k-3$; say it equals $\partial_{\Delta} \beta$. Then defining $\psi\left(s^{-1} \omega^{n-p-t} v_{i j}^{*}\right)=\beta$ gives an extension of $\psi$ with the required property. This proves the claim.

This extends $\psi$ over all elements of $s^{-1} H_{*}$ of degree $\leq\left|s^{-1} \omega^{p}\right|$. Finally, define $\psi$ to be zero on all elements $s^{-1} \omega^{m}$ for $m>p$. This completes the definition of $\psi$.

According to 4.4 , any such map $\Phi=1+\psi$ defines an isomorphic perturbation $\mathbf{L}\left(s^{-1} H_{*}, \partial_{\Delta}+Q\right)$ with

$$
Q=P+\psi \partial_{\Delta}+\psi P-\partial_{\Delta} \psi-Q \psi .
$$

Claim. The perturbation so defined has $Q_{3}$ equal to zero on all elements of degree $\leq\left|s^{-1} \omega^{p}\right|$, and is zero on elements of degree $\leq n+k-2$.

Proof of claim. The length three part of $Q$ is given by

$$
Q_{3}=P_{3}+\psi \partial_{\Delta}-\partial_{\Delta} \psi
$$

This is trivially zero on all elements of degree lower than $\left|s^{-1} \omega^{n-p} V_{i}^{*}\right|$ in the column of $V_{i}$, for each $i$. On elements of $s^{-1} \omega^{n-p} V_{i}^{*}$, for each $i, P_{3}=0-$ since these are of degree $<\left|s^{-1} \omega^{p}\right|, \psi \partial_{\Delta}=0$ - since $\partial_{\Delta}\left(s^{-1} \omega^{n-p} v_{i j}^{*}\right)$ is a sum of terms whose entries are of degree $<n-1$, and $\psi\left(s^{-1} \omega^{n-p} v_{i j}^{*}\right)= \pm X_{i j}$, so $\partial_{\Delta} \psi=0$ on these elements also. Hence $Q_{3}=0$ on elements of $s^{-1} \omega^{n-p} V_{i}^{*}$, for each $i$. Next, on elements of $s^{-1} \omega^{n-p-t} V_{i}^{*}$ for $t>0, P_{3}$ is zero, by assumption, and $\psi \partial_{\Delta}-\partial_{\Delta} \psi=0$ by the construction of the previous claim. So $Q_{3}=0$ on all these elements also. On elements of type $s^{-1} \omega^{r}$ for $r<p, P_{3}$ is zero, $\psi$ is zero by definition and, for dimensional reasons, no elements of $s^{-1} \omega^{n-p-t} V_{i}^{*}$ for $t \geq 0$ can appear in the formula for $\partial_{\Delta} s^{-1} \omega^{r}$. Hence $\psi \partial_{\Delta}$ will be zero here and so $Q_{3}$ equals zero on all these elements also. Finally, on $s^{-1} \omega^{p}$, again no elements of $s^{-1} \omega^{n-p-t} V_{i}^{*}$ for $t>0$ can appear in the formula for $\partial_{\Delta} s^{-1} \omega^{p}$, so $\psi \partial_{\Delta}$ remains what it was originally on this element. 
Thus, by construction, $Q_{3}=0$ on this element too. It is easy to check, in a similar fashion, that $Q$ is zero on elements of degree $\leq n+k-2$. This proves the claim.

An induction argument on $p \leq n$ now completes the proof of the proposition. Q.E.D.

It is now possible to give an outline:

6.6. Proof of Theorem 2. Let $H=H(n, k)$ be a Lefschetz algebra with $2 n \leq$ $4 k-2$, and $\mathbf{L}\left(s^{-1} H_{*}, \partial_{\Delta}+P\right)$ be any perturbation of the Quillen model of $H$. Corollary 6.3 and Proposition 6.5 above imply that it is possible to assume, without loss of generality, that $P_{3}=0$. Now the information given by Proposition 6.2 above, a slight modification of 4.4, and standard arguments for removing perturbations (such as those of $\left[\mathrm{Mi}_{2}\right]$ or $[\mathrm{St}]$ ) make it possible to remove first the length four part of the perturbation, and subsequently all of the remaining perturbation. For details see my thesis [Lu].

\section{EXAMPLES AND FINAL REMARKS}

In this final section, some relatively simple examples of Lefschetz algebras are considered. As mentioned in the introduction to $\S 5$, many of these will fit into the hypotheses of Theorem 1 above. Also, two examples of Lefschetz algebras that are not intrinsically formal are given; one of which (7.9) is the cohomology of a Kähler manifold. Finally, two remarks are made concerning extensions of the results in this paper.

7.1. Example. The simplest type of Lefschetz algebra, other than the trivial algebra, is a truncated polynomial ring on one generator of degree $2, \mathbb{Q}[x] /\left(x^{n+1}\right)$. Such an algebra is realised as the cohomology of $n$-dimensional complex projective space, $H^{*}\left(\mathbb{C} P^{n} ; \mathbb{Q}\right)$, that is, the cohomology ring of a Kähler manifold. These algebras are (elliptic graded) complete intersections and so are intrinsically formal, as is well known.

7.2. Example. Let $G(k, n)$ be the complex Grassmannian manifold of $k$ dimensional subspaces in $(n+k)$-dimensional space. Then $H^{*}(G(k, n) ; \mathbb{Q}) \cong$ $\mathbb{Q}\left[x_{1}, x_{2}, \ldots, x_{k}, y_{1}, \ldots, y_{n}\right] /\left(R_{1}, \ldots, R_{n+k}\right)$; where $\left|x_{i}\right|=2 i=\left|y_{i}\right|,\left|R_{i}\right|=$ $2 i$, and $R_{i}$ is the homogeneous degree $2 i$ part of the equation $\left(1+x_{1}+\cdots+\right.$ $\left.x_{k}\right)\left(y_{1}+\cdots+y_{n}\right)=1$. As in [Bott-Tu, p. 297], $H^{*}(G(k, n) ; \mathbb{Q})$ is an (elliptic) complete intersection and hence is intrinsically formal. Furthermore, $H^{*}(G(k, n) ; \mathbb{Q})$ is a Lefschetz algebra since by construction it is realised by a Kähler manifold.

7.3. Construction. Consider the following construction [Bott-Tu, IV, §2.1]. Let $p: E \rightarrow M$ be a complex vector bundle of rank $n$, and let $\pi: P(E) \rightarrow M$ be the projective bundle. Then if $\pi^{-1} E \rightarrow P(E)$ is the pullback bundle over $P(E)$, $\pi^{-1} E$ is a rank $n$ bundle, which splits as the direct sum of a complex line bundle and a rank $n-1$ bundle; say $\pi^{-1} E \cong S_{1} \oplus Q_{1}$. In order to construct 
a split manifold for $p$, this construction can be repeated $n-1$ times. In the special case when $p$ is the trivial bundle over a point, $p: \mathbb{C}^{n} \rightarrow *$, this iterated construction results in the flag manifold $\mathrm{Fl}(n)$, and a sequence of manifolds

$$
\mathrm{Fl}(n)=P\left(Q_{n, n-2}\right) \rightarrow \cdots \rightarrow P\left(Q_{n, 1}\right) \rightarrow \mathbb{C} P^{n} \rightarrow * .
$$

An attractive feature of these examples is the fact that there is a presentation of the cohomology rings of these manifolds, given by

$$
H^{*}\left(P\left(Q_{n, i}\right) ; \mathbb{Q}\right) \cong \mathbb{Q}\left[x_{1}, \ldots, x_{i+1}, y_{1}, \ldots, y_{n-i-1}\right] /\left(R_{1}, \ldots, R_{n}\right),
$$

where $\left|x_{j}\right|=2,\left|y_{j}\right|=2 j,\left|R_{j}\right|=2 j$, and $R_{j}$ is the homogeneous degree $2 j$ part of the equation $\left(1+x_{1}\right) \cdots\left(1+x_{i+1}\right)\left(1+y_{1}+\cdots+y_{n-i-1}\right)=1$. To complete the notation, $\mathbb{C} P^{n}$ should be regarded as $P\left(Q_{n, 0}\right)$.

7.4. Lemma. With the above notation, $H^{*}\left(P\left(Q_{i, n}\right) ; \mathbb{Q}\right)$ is a Lefschetz algebra and an elliptic graded complete intersection.

Proof. That $H^{*}\left(P\left(Q_{i, n}\right) ; \mathbb{Q}\right)$ is a graded complete intersection follows from an easy generalisation of the argument in [Bott-Tu, p. 297], to show that the relations form a regular sequence. Furthermore, each $P\left(Q_{i, n}\right)$ is actually a Kähler manifold. This follows since $P\left(Q_{i, n}\right)$ is a generalised flag manifold, and a similar argument to [W, p. 11] gives an embedding of $P\left(Q_{i, n}\right)$ into some $\mathbb{C} P^{n}$; finally, any complex manifold that admits such an embedding is Kähler. Q.E.D.

7.5. Examples. The above construction together with 7.1 and 7.2 provides many examples of algebras that, from the intrinsic formality point of view, are the simplest cases of Lefschetz algebras-from the intrinsic formality point of view, the simplest algebras are free algebras, and these cannot be Lefschetz. They are all (elliptic) complete intersections and hence are intrinsically formal. In addition, these examples may be combined to give even more simplest cases, by forming the tensor product, since the properties of being Lefschetz and of being a complete intersection are both preserved under tensor product. For these examples, all tensor products can in fact be realised by Kähler manifolds, as the product of two Kähler manifolds is again a Kähler manifold.

Clearly the above examples can be used to construct plenty of algebras fitting the hypotheses of Theorem 1 , since it is easy to construct $(n-1)$-connected Poincaré duality algebras of dimension $2 n$. However, as a more natural example of an algebra to which Theorem 1 applies, there is the following.

7.6. Example. Consider the cohomology ring $H^{*}(X ; \mathbb{Q})$, of a complete intersection $X$ [Hir, p. 159], not to be confused with the algebraic complete intersections previously mentioned. These cases have been considered in [Bab], where it is shown that these algebras are intrinsically formal. They have also been considered in $\left[\mathrm{N}_{1}\right]$, where the rational homotopy of spaces realising such algebras is completely calculated. No explicit mention of intrinsic formality is made in $\left[\mathrm{N}_{1}\right]$, although the intrinsic formality of $H^{*}(X ; \mathbb{Q})$ could be more 
or less read off from that work. In any case, if $X$ is a complete intersection, $H^{*}(X ; \mathbb{Q})$ is isomorphic to a connected sum of $H^{*}\left(\mathbb{C} P^{n} ; \mathbb{Q}\right)$, some $n$, and an $(n-1)$-connected Poincaré duality algebra of dimension $2 n$. So by Theorem $1, H^{*}(X ; \mathbb{Q})$ is intrinsically formal.

7.7. Corollary. Let $X$ be a complete intersection; then $X$ is formal.

Remark. This gives an alternative proof of a rather special case of the theorem that Kähler manifolds are formal. Whilst 7.7 is hardly a substantial part of this theorem, at least the result is valid over the rationals directly; whereas the result of [D-Gr-Mo-Su] needs a theorem of descent before applying over the rationals.

This latter example should be thought of as the next hardest case after $H^{*}\left(\mathbb{C} P^{n} ; \mathbb{Q}\right)$ and examples such as those from 7.1-7.5 above. It was this example that prompted the final generality of Theorem 1. Thus, in the above examples, the "easiest cases" of Lefschetz algebras are by definition intrinsically formal, and Theorem 1 should be thought of as taking up the next hardest case.

The last two examples given are of Lefschetz algebras that are not intrinsically formal. The second of these is the cohomology of a Kähler manifold, and so shows that Kähler manifolds do not, in general, have intrinsically formal cohomology rings. This fact is considered in [Bab], and an example given there; however there is an error in the argument, and the algebra given in [Bab], and claimed not to be intrinsically formal, is, in fact, intrinsically formal. My example is a minor modification of Babenko's. The first of the examples is one that lies immediately outside the dimensional restrictions of Theorem 2 , and so shows that result-within the terms set up there-to be best possible. I do not know whether it is realised by a Kähler manifold, and I do not know whether there are Kähler manifolds whose cohomology lies immediately outside the range of Theorem 2 .

7.8. Example. Let $X=\left\{\left\{\left\{\mathbb{C} P^{2} \#\left(\mathbf{S}^{2} \times \mathbf{S}^{2}\right)\right\} \times \mathbb{C} P^{1}\right\} \#\left(\mathbf{S}^{3} \times \mathbf{S}^{3}\right)\right\} \times \mathbb{C} P^{1} . \mathbf{S}^{2}$ and $\mathbb{C} P^{n}$ are Kähler manifolds, and so repeated applications of $3.6,3.8$ and 3.9 give that $H^{*}(X ; \mathbb{Q})$ is a Lefschetz algebra. Now, $H^{*}(X ; \mathbb{Q})$ can be presented as $\Lambda\left(\omega_{1}, \omega_{2}, \omega_{3}, a, b, \alpha, \beta\right) /(R)$, where the $\omega_{i}$ 's are representatives for the $\mathbb{C} P^{n}$,s and $a, b, \alpha, \beta$ for the $S^{i}$,s-so $\left|\omega_{i}\right|=2,|a|=|b|=2$, and $|\alpha|=$ $|\beta|=3$; and $R=\left\{a^{2}, b^{2}, a b-\omega_{1}^{2}, \omega_{1} a, \omega_{1} b, \omega_{2}^{2}, \omega_{3}^{2}, \alpha a, \beta a, \alpha b, \beta b, \alpha \beta-\right.$ $\left.\omega_{1}^{2} \omega_{2}, \alpha \omega_{1}, \beta \omega_{1}, \alpha \omega_{2}, \beta \omega_{2}\right\}$. Using this presentation, it is possible to construct the differential in $\mathbf{L}\left(s^{-1} H_{*}(X ; \mathbb{Q}), \partial_{\Delta}\right)$. Furthermore, it is possible to check that the following defines a perturbation on $\mathbf{L}\left(s^{-1} H_{*}(X ; \mathbb{Q}), \partial_{\Delta}\right)$, and that the resulting $\mathbf{L}\left(s^{-1} H_{*}(X ; \mathbb{Q}), \partial_{\Delta}+P\right)$ is not isomorphic to $\mathbf{L}\left(s^{-1} H_{*}(X ; \mathbb{Q}), \partial_{\Delta}\right)$ :

$$
\begin{gathered}
P\left(s^{-1} \omega_{3} \alpha\right)=-\left[s^{-1} \omega_{1},\left[s^{-1} a, s^{-1} a\right]\right], \\
P\left(s^{-1} \omega_{3} \omega_{2} \omega_{1}\right)=-\left[s^{-1} \beta,\left[s^{-1} a, s^{-1} a\right]\right],
\end{gathered}
$$




$$
P\left(s^{-1} \omega_{3} \omega_{2} b\right)=2\left[s^{-1} a,\left[s^{-1} \beta, s^{-1} \omega_{1}\right]\right]
$$

and $P=0$ on all other elements of $s^{-1} H_{*}(X ; \mathbb{Q})$. Details are in my thesis $[\mathrm{Lu}]$. Hence, $H^{*}(X ; \mathbb{Q})$ is not intrinsically formal.

7.9. Example. Let $V_{3}^{(3)}$ be a hypersurface of dimension 3 in $\mathbb{C} P^{4}$, defined by a single equation of degree 3. A good description of the cohomology of such spaces is given in [Bab]; see also [Hir, p. 159]. $H^{*}\left(V_{3}^{(3)} ; \mathbb{Q}\right)$ has algebra generators $\omega$ in degree $2,\left\{a_{i}, a_{i}^{*}\right\}_{i=1, \ldots, 5}$ in degree 3 , and is isomorphic to $H^{*}\left(\mathbb{C} P^{3} \#\left(\mathbf{S}^{3} \times \mathbf{S}^{3}\right) \# \cdots \#\left(\mathbf{S}^{3} \times \mathbf{S}^{3}\right) ; \mathbb{Q}\right)$. Now consider $X=V_{3}^{(3)} \times$ $\mathbb{C} P^{2}: X$ is a Kähler manifold since both factors are. However, $H^{*}(X ; \mathbb{Q})$ is not intrinsically formal, as the following defines a nontrivial perturbation of $\mathbf{L}\left(s^{-1} H_{*}(X ; \mathbb{Q}), \partial_{\Delta}\right)$. Let $x$ generate the cohomology of $\mathbb{C} P^{2} ;$ then define

$$
\begin{gathered}
P\left(s^{-1} a_{3} x^{2}\right)=-\left[s^{-1} \omega,\left[s^{-1} a_{1}, s^{-1} a_{2}\right]\right], \\
P\left(s^{-1} \omega^{2} x^{2}\right)=-\left[s^{-1} a_{3}^{*},\left[s^{-1} a_{1}, s^{-1} a_{2}\right]\right], \\
P\left(s^{-1} a_{1}^{*} x^{2}\right)=\left[s^{-1} a_{2},\left[s^{-1} a_{3}^{*}, s^{-1} \omega\right]\right]
\end{gathered}
$$

and

$$
P\left(s^{-1} a_{2}^{*} x^{2}\right)=-\left[s^{-1} a_{1},\left[s^{-1} a_{3}^{*}, s^{-1} \omega\right]\right] .
$$

Then it is possible to check that, so defined, $\partial_{\Delta}+P$ is a differential on $\mathbf{L}\left(s^{-1} H_{*}(X ; \mathbb{Q})\right)$. In fact, the only nontrivial checking to be done is to check that $P \partial_{\Delta}\left(s^{-1} \omega^{3} x^{2}\right)=0$, and with the help of the Jacobi identity, this is true by construction. Finally, it remains to show that $\mathbf{L}\left(s^{-1} H_{*}(X ; \mathbb{Q}), \partial_{\Delta}+P\right)$ cannot be isomorphic to $\mathbf{L}\left(s^{-1} H_{*}(X ; \mathbb{Q}), \partial_{\Delta}\right)$. First, it is the case that any isomorphism

$$
\Phi: \mathbf{L}\left(s^{-1} H_{*}(X ; \mathbb{Q}), \partial_{\Delta}+P\right) \rightarrow \mathbf{L}\left(s^{-1} H_{*}(X ; \mathbb{Q}), \partial_{\Delta}\right)
$$

can be used to define an isomorphism

$$
1+\psi: \mathbf{L}\left(s^{-1} H_{*}(X ; \mathbb{Q}), \partial_{\Delta}+P\right) \rightarrow \mathbf{L}\left(s^{-1} H_{*}(X ; \mathbb{Q}), \partial_{\Delta}\right),
$$

where $\psi$ increases bracket length by at least one. So without loss of generality assume there is an isomorphism

$$
\Phi=1+\psi: \mathbf{L}\left(s^{-1} H_{*}(X ; \mathbb{Q}), \partial_{\Delta}+P\right) \rightarrow \mathbf{L}\left(s^{-1} H_{*}(X ; \mathbb{Q}), \partial_{\Delta}\right) .
$$

Then $\Phi\left(\partial_{\Delta}+P\right)\left(s^{-1} a_{3} x^{2}\right)$ must equal $\partial_{\Delta} \Phi\left(s^{-1} a_{3} x^{2}\right)$. But this implies that, modulo length greater than 3 terms, and the terms in the (Lie) ideal generated by $s^{-1} a_{3}$ and $s^{-1} x,\left[s^{-1} \omega,\left[s^{-1} a_{1}, s^{-1} a_{2}\right]\right]$ is a boundary, which cannot be true since, modulo these terms, the image of $\partial_{\Delta}$ is contained in the ideal generated by $\left[s^{-1} \omega, s^{-1} \omega\right],\left[s^{-1} \omega, s^{-1} \omega^{2}\right]$, and $\left[s^{-1} a_{i}, s^{-1} a_{i}^{*}\right]$. Thus, there cannot exist an isomorphism between $\mathbf{L}\left(s^{-1} H_{*}(X ; \mathbb{Q}), \partial_{\Delta}+P\right)$ and $\mathbf{L}\left(s^{-1} H_{*}(X ; \mathbb{Q}), \partial_{\Delta}\right)$, so $H^{*}(X ; \mathbb{Q})$ cannot be intrinsically formal.

Remark. In 3.1, Lefschetz algebras were defined with Kähler classes in degree 2. The reason for this is, as explained in $\S \S 1,2$, and 3 , that the motivation for the 
work originally came from the problem of deciding the intrinsic formality or not of the cohomology of Kähler manifolds. However, it would be possible to set up, in an obvious way, a more general type of algebra with a "Kähler class" in degree $2 k$, for any $k \geq 1$. With this generalised definition, all the (purely algebraic) working of, for example, Theorems 1 and 2, would go through in a formally identical way.

Remark. Theorem 1 is not best possible as it stands, and there is a generalisation. An identical argument to that given in my thesis [Lu] to prove 4.3.9 there gives the following: Let $H$ be a Poincaré duality algebra of dimension $2 n$ such that there exists an inclusion of algebras $i: G \rightarrow H$ with $G$ an elliptic complete intersection of degree $2 n$. If $i$ is a bijection in degrees $\leq n-2$, then $H$ is intrinsically formal. As in Theorem 1, the algebras under consideration need not be Lefschetz. However, again as in Theorem 1, the examples of 7.1-7.5 can be used to provide examples of Lefschetz algebras that fit the hypotheses of this result. The example of 7.8 above lies immediately outside this dimension restriction and so shows that this result-within the terms set up here-is best possible.

\section{BIBLIOGRAPHY}

[Bab] I. K. Babenko, On real homotopy properties of complete intersections, Math. USSR Izv. 15 (1980), 241-258.

[Ba-Le] H. J. Baues and J.-M. Lemaire, Minimal models in homotopy theory, Math. Ann. 225 (1977), 219-242.

[Bor] A. Borel, Seminar on transformation groups, Ann. of Math. Stud., no. 46, Princeton Univ. Press, Princeton, N.J., 1960.

[Bott-Tu] R. Bott and L. Tu, Differential forms in algebraic topology, Graduate Texts in Math., vol. 82, Springer-Verlag, 1982.

[Bou-Gu] A. K. Bousfield and V. K. A. M. Guggenheim, On PL de Rham theory and rational homotopy theory, Mem. Amer. Math. Soc. No. 179 (1976).

[D-Gr-Mo-Su] P. Deligne, P. A. Griffiths, J. Morgan, and D. Sullivan, Real homotopy theory of Kähler manifolds, Invent. Math. 29 (1975), 245-274.

[Fé-H] Y. Félix and S. Halperin, Formal spaces with finite dimensional rational homotopy, Trans. Amer. Math. Soc. 270 (1982), 575-588.

[Fé-H-Th] Y. Félix, S. Halperin, and J.-C. Thomas, The homotopy Lie algebra for finite complexes, Inst. Hautes Études Sci. Publ. Math. 56 (1982), 179-202.

[Fr-H] J. B. Friedlander and S. Halperin, An arithmetic characterization of the rational homotopy groups of certain spaces, Invent. Math. 53 (1979), 117-133.

[Gr-Mo] P. A. Griffiths and J. Morgan, Rational homotopy theory and differential forms, Progr. in Math., vol. 16, Birkhäuser, 1981.

$\left[\mathrm{H}_{1}\right]$ S. Halperin, Lectures on minimal models, Mém. Soc. Math. France (N.S.) 9-10 (1983).

$\left[\mathrm{H}_{2}\right]-$ Finiteness in the minimal models of Sullivan, Trans. Amer. Math. Soc. 230 (1977), 173-199.

[H-St] S. Halperin and J. Stasheff, Obstructions to homotopy equivalences, Adv. in Math. 32 (1979), 233-279.

[Hir] F. Hirzebruch, Topological methods in algebraic geometry, Springer-Verlag, 1966.

[Józ] T. Józefiak, A homological characterisation of graded complete intersections. I, J. Pure Appl. Algebra 8 (1976), 143-148. 
[Le-Si] J.-M. Lemaire and F. Sigrist, Sur les invariants d'homotopie rationelle liés à la L. S. catégorie, Comment. Math. Helv. 56 (1981), 103-122.

[Lu] G. M. Lupton, The intrinsic formality of certain types of algebras, Ph. D. Thesis, University of Edinburgh, 1986.

[Mac] R. MacPherson, Global questions in the topology of singular spaces, Proc. Internat. Congr. Math. (Warsaw, 1983), PWN, Warsaw, 1984, pp. 213-235.

[Me] W. Meier, Some topological properties of Kähler manifolds and homogeneous spaces, Math. Z. 183 (1983), 473-481.

$\left[\mathrm{Mi}_{1}\right]$ T. J. Miller, Rational homotopy and minimal Lie algebras, Ph. D. Thesis, Univ. of Notre Dame, 1976.

$\left[\mathrm{Mi}_{2}\right] \_$, On the formality of $(k-1)$-connected compact manifolds of dimension less than or equal to $4 k-2$, Illinois J. Math. 23 (1979), 253-258.

$\left[\mathrm{N}_{1}\right]$ J. Neisendorfer, The rational homotopy groups of complete intersections, Illinois J. Math. 23 (1979), 175-182.

$\left[\mathrm{N}_{2}\right] \_$, Lie algebras, coalgebras and rational homotopy theory for nilpotent spaces, Pacific J. Math. 74 (1978), 429-460.

[N-Mi] J. Neisendorfer and T. J. Miller, Formal and coformal spaces, Illinois J. Math. 22 (1978), 565-580.

[Q] D. Quillen, Rational homotopy theory, Ann. of Math. (2) 90 (1969), 205-295.

[St] J. Stasheff, Rational Poincaré duality spaces, Illinois J. Math. 27 (1983), 104-109.

$\left[\mathrm{Su}_{1}\right]$ D. Sullivan, Differential forms and the topology of manifolds, Proc. Conf. on Manifolds, (Tokyo, 1973).

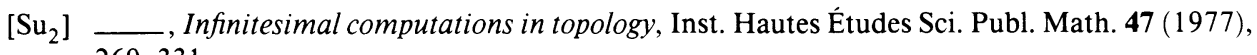
269-331.

[Ta] D. Tanré, Cohomologie de Harrison et espaces projectifs Tronqués, J. Pure Appl. Algebra 38 (1985), 353-366.

[W] R. O. Wells, Differential analysis on complex manifolds, Graduate Texts in Math., vol. 65, Springer-Verlag, 1980.

Department of Mathematics, Dartmouth College, Hanover, New Hampshire 03755 